\title{
Activation of the alternative complement pathway: clinical application of a new technique to measure fragment $\mathrm{Ba}$
}

\author{
G SENALDi, M PEAKMAN, A ALHAQ, V A MAKINDE, D E H TEE, D VERGANi \\ From the Department of Immunology, King's College School of Medicine and Dentistry, London
}

SUMMARY A new laser nephelometric technique for the measurement of the alternative complement pathway fragment $\mathrm{Ba}$ has been developed. Activation of the alternative complement pathway was assessed in 16 patients with Gram negative bacteraemia, six with Gram positive bacteraemia, 20 with rheumatoid arthritis, and 18 healthy subjects. Patients with Gram negative bacteraemia had significantly higher values of $\mathrm{Ba}$ (median $14.8 \%)$ than controls $(9.3 \%)(\mathrm{p}<0.01)$, while patients with Gram positive bacteraemia and rheumatoid arthritis had values similar to those of controls (10.1\% and $9.5 \%)$. The technique proved sensitive and precise, and is suitable for the routine laboratory evaluation of complement activation through the alternative pathway.

Activation of the complement system has an important role in the pathogenesis of numerous immunologically mediated disorders. ${ }^{1-3}$ The complement cascade is activated through two major pathways (fig 1). The alternative pathway is activated by complex polysaccharides of Gram negative bacteria, while the classical pathway is triggered by immune complexes. ${ }^{4}$ Both pathways converge at the level of $\mathrm{C} 3$ to progress in a common sequence leading to the formation of the membrane attack complex. Alternative pathway activation results in the splitting of factor $\mathrm{B}$ into a large $\mathrm{Bb}$ molecule (molecular weight 60000 ) and a smaller fragment $\mathrm{Ba}$ (molecular weight $33000)$. $\mathrm{Bb}$ contributes to the formation of the alternative pathway $\mathrm{C} 3$ convertase $(\mathrm{C} 3 \mathrm{bBb}) ; \mathrm{Ba}$ is physiologically inactive and takes no further part in the complement cascade. ${ }^{5}$ Increased concentrations of $\mathrm{Ba}$ thus provide a specific index of ongoing activation of the alternative complement pathway. In Gram negative bacterial infections complement is activated by direct contact with the cell wall of micro-organisms. ${ }^{6}$ Quantitation of complement activation via the alternative pathway may provide a useful tool for evaluating the progression and severity of Gram negative infections. ${ }^{7}$ In rheumatoid arthritis, a typical immune complex disease,${ }^{8}$ complement is thought to be activated through the classical pathway, ${ }^{9}$ though activa-

Accepted for publication 22 April 1987 tion through the alternative route has been reported. ${ }^{10}$

In this study we show how Ba can be separated from the larger factor B related molecules by precip-

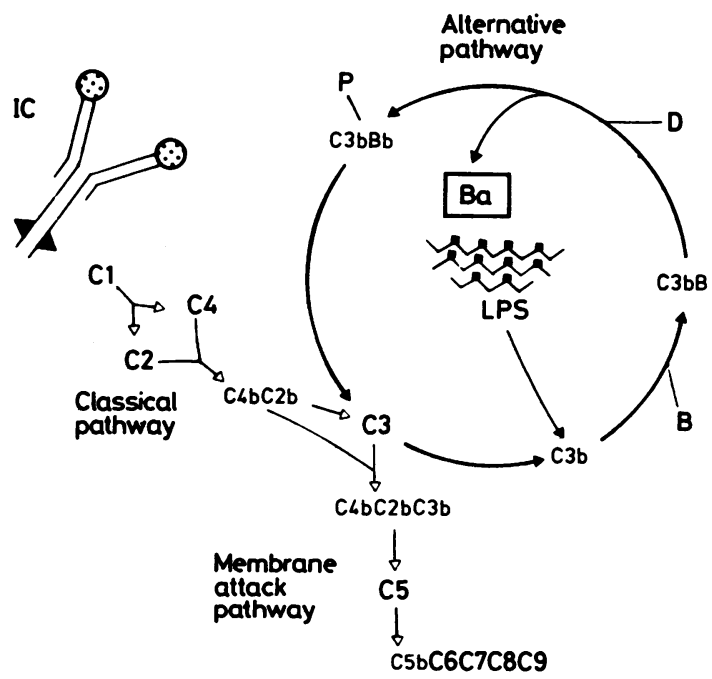

Fig 1 Diagram of the pathways of complement activation. Alternative pathway is activated by lipopolysaccharides (LPS) and the classical by immune complexes (IC). Fragment $\mathrm{Ba}$ is generated from factor $\mathrm{B}$ during activation of alternative complement pathway. 
itation with polyethylene glycol. Ba can then be measured by a laser nephelometric technique, using an antiserum reacting with determinants present on the whole molecule of factor B. We analysed activation of the alternative complement pathway in groups of patients with Gram negative bacteraemia (GNB), Gram positive bacteraemia (GPB), rheumatoid arthritis and in healthy subjects.

\section{Material and methods}

\section{CLINICAL SAMPLES}

Blood collected by venepuncture into a final edectic acid concentration of $10 \mathrm{mmol} / \mathrm{l}$ was immediately separated by centrifugation at $1000 \mathrm{~g}$ at $4^{\circ} \mathrm{C}$ for 15 minutes and the plasma stored at $-70^{\circ} \mathrm{C}$. Samples were obtained from 16 patients (nine men, median age 46 years, range 17-72) with blood samples positive on culture for Gram negative bacteria; six patients (three men, median age 34 years, range 1-70) with blood samples positive on culture for Gram positive bacteria, 20 patients (five men, median age 52 years, range 27-71) with "classical" or "definite" rheumatoid arthritis according to the criteria of the American Rheumatism Association, and 18 healthy subjects (nine men, median age 32 , range $27-47$ ). The degree of disease activity in patients with rheumatoid arthritis was assessed by measuring the erythrocyte sedimentation rate (ESR) $(\mathrm{mm} /$ first hour).

FRAGMENT Ba STANDARD

Normal human serum was incubated at $37^{\circ} \mathrm{C}$ for 60 minutes with inulin (BDH Biochemicals, Poole, Dorset, England) at a concentration of $3 \mathrm{mg} / \mathrm{ml}$ to activate exhaustively complement through the alternative pathway. ${ }^{11}$ After centrifugation at $1500 \mathrm{~g}$ for 30 minutes the supernatant was frozen in aliquots and stored at $-70^{\circ} \mathrm{C}$. This $\mathrm{Ba}$ enriched preparation was used throughout the study as the source of the $\mathrm{Ba}$ standard.

To separate fragment $\mathrm{Ba}$ from native factor $\mathrm{B}$ and fragment $\mathrm{Bb} 100 \mu \mathrm{l}$ of $\mathrm{Ba}$ standard were added to $100 \mu \mathrm{l}$ of polyethylene glycol 6000 (PEG) (Sigma Chemicals, Poole, Dorset, England) in Dulbecco's

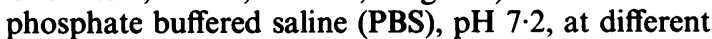
final concentrations ranging from $2-24 \%$, incubated for 90 minutes at $4^{\circ} \mathrm{C}$, and centrifuged at $1500 \mathrm{~g}$ at $4^{\circ} \mathrm{C}$ for 30 minutes. This aims to precipitate the larger factor $\mathrm{B}$ and $\mathrm{Bb}$, leaving $\mathrm{Ba}$ in the supernatant. ${ }^{12}$ The efficacy of various PEG concentrations in separating $\mathrm{Ba}$ was assessed by a double dimension immunodiffusion performed in $1 \%$ agarose $15(\mathrm{BDH}$ Biochemicals) in PBS containing 2\% PEG.

To establish the working conditions of the assay, a checker board titration was performed. Supernatant from $\mathrm{Ba}$ standard treated with PEG was serially diluted in PBS and incubated at room temperature with different concentrations of anti-factor B anti- 7 serum (Dakopatts, High Wycombe, Buckinghamshire, England) in PBS. Readings were taken $\frac{\text { - }}{-}$ in a Behring laser nephelometer at $15,30,45,60,90$, 120,150 and 180 minutes and referred to a reference curve prepared from Ba standard.

Statistical analysis was carried out using the Wilcoxon's rank sum test and the Spearman's rank regression analysis.

\section{Results}

Ba was successfully separated from larger factor $\mathbf{B} \overrightarrow{\vec{\omega}}$ related molecules by precipitation in a final concen- $\frac{\sigma}{2}$ tration of PEG of $18 \%$ (fig 2). At this concentration of PEG larger factor $B$ related molecules were precip- $\overrightarrow{0}$ itated and the smaller fragment $\mathrm{Ba}$ remained in the $\overrightarrow{-}$ supernatant. Higher concentrations of PEG tended to precipitate $\mathrm{Ba}$, while lower concentrations did not $\underset{\mathrm{W}}{\vec{N}}$ completely remove larger factor B related molecules o from the supernatant.

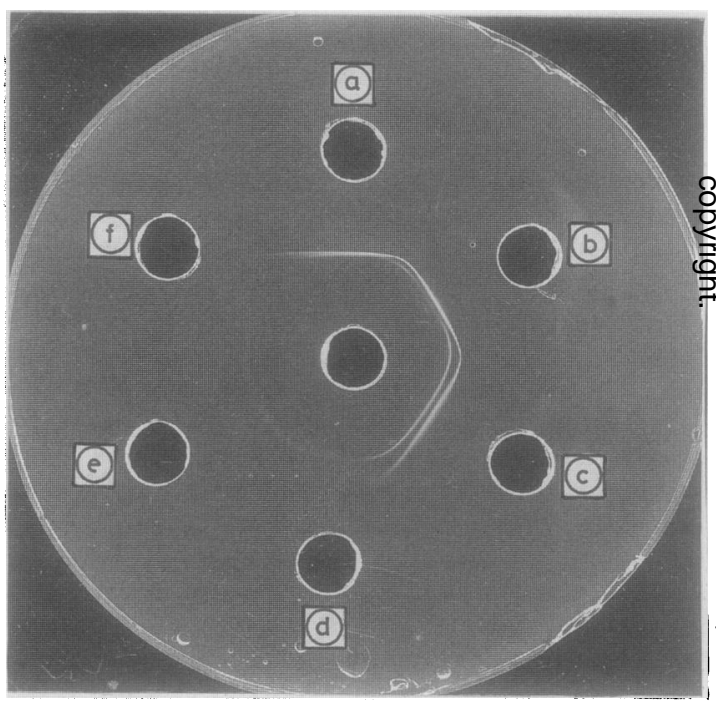

Fig 2 Ba separation assessed by double dimension immunodiffusion.

Central well: neat anti-factor $B$ antiserum. Well $A$ : edetic acid plasma; well $B$; normal human serum (NHS); well $C$ $B a$ standard (NHS treated with inulin). Wells $D, E, F$ : supernatants recovered after PEG treatment of Ba standard at final concentrations of $16 \%, 18 \%$, and $20 \%$,

respectively.

Anti-factor $B$ antiserum gives two fused precipitin arcs, when reacting with normal edetic acid plasma $(a)$. The two precipitin lines become more apparent in NHS $(b)$, and are completely distinct when NHS is treated with inulin $(c)$.

The heavier of the two fragments is successfully removed by treatment with PEG at a final concentration of $16 \%$ (d), $18 \%(e)$, and $20 \%(f)$. 
MEASUREMENT OF FRAGMENT Ba BY LASER NEPHELOMETRY

$\mathrm{Ba}$ was separated by treating $\mathrm{Ba}$ standard and test samples with $18 \%$ PEG. The optimal conditions for the measurement of $\mathrm{Ba}$ by laser nephelometry were as follows. Supernatants from Ba standard and test samples diluted $1 / 4$ in PBS were added to an equal volume of anti-factor B antiserum diluted $1 / 5$ in PBS. After incubation for 120 minutes at room temperature the voltage was read in a laser nephelometer. The top point of the reference curve was obtained by diluting the $100 \%$ Ba standard containing $18 \%$ PEG $1 / 4$ in PBS. This first dilution led to a PEG concentration of $4.5 \%$. The remaining points of the reference curve were derived by double dilution of the 1/4 top standard to $1 / 64$ in $4.5 \%$ PEG in PBS. These dilutions were chosen because a linear relation between voltage and $\mathrm{Ba}$ concentration in this range was found (fig 3 ). Thus after anti-serum had been added all dilutions of supernatants resulted in a final PEG concentration of $2 \cdot 25 \%$. At this PEG concentration optimum discrimination of voltage for different concentrations of $\mathbf{B a}$ occurred. Diluting supernatants from test samples diluted $1 / 4$ in PBS allowed results to be read within the straight part of the reference curve. Varying the incubation time from 15 to 180 minutes before reading the voltage on the laser nephelometer showed that stable voltage was obtained at 120 minutes. This was therefore chosen as the optimum period of incubation.

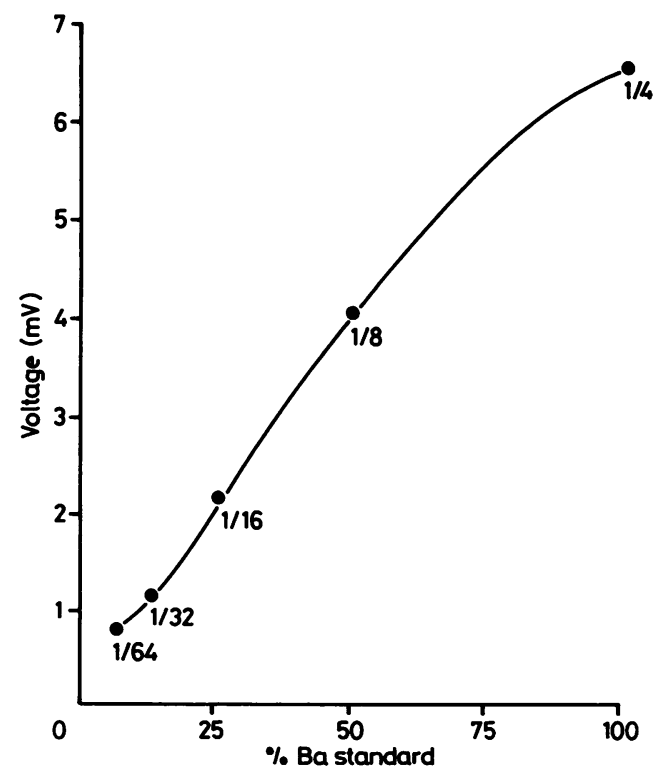

Fig 3 Reference curve derived from serial dilutions of the supernatant of Ba standard from $1 / 4$ to $1 / 64$. The top dilution is given a value of $100 \%$.
The intra-assay coefficient of variation derived from estimating the same sample 30 times was $4 \cdot 6 \%$. This interassay coefficient of variation obtained by repeating the evaluation of the same sample on 10 different occasions was $13 \cdot 8 \%$.

Fig 4 shows the values in 16 patients with GNB, six with GPB, 20 with rheumatoid arthritis and in 18 healthy subjects. Ba values are expressed as a percentage of the top standard, which is regarded as $100 \%$. Low yet measurable Ba values were present in all the normal subjects tested. Patients with GNB had significantly higher values of $\mathrm{Ba}$ (median $14.8 \%$, range $7 \cdot 0-38.3 \%$ ) compared with controls $(9.3 \%$, $6.5-14.4 \% ; \mathrm{p}<0.01)$. Nine $(56 \%)$ had $\mathrm{Ba}$ values in excess of the upper limit of normal. Patients with GPB and with rheumatoid arthritis showed $B a$ values $(10 \cdot 1 \%, 6 \cdot 1-12 \cdot 7 \%$ and $9 \cdot 5 \%, 4-17 \cdot 4 \%$, respectively) not significantly different from those of controls and significantly lower than those in patients with GNB $(\mathrm{p}<0.01)$. Four patients with rheumatoid arthritis had Ba values in excess of the upper limit of normal. These patients had active disease (ESR $>40 \mathrm{~mm}$ first hour), and in patients with rheumatoid arthritis a

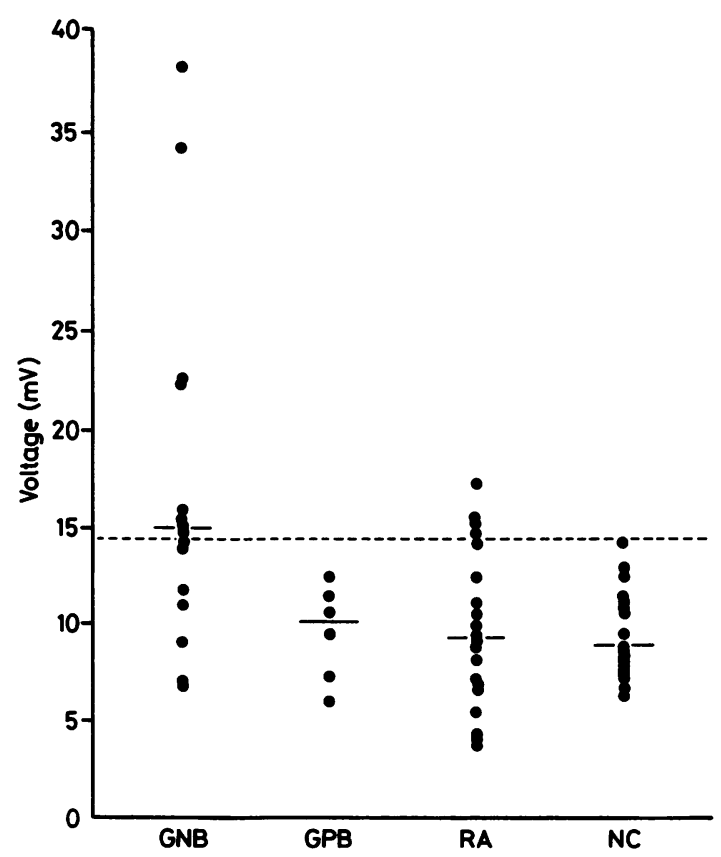

Fig. $4 \mathrm{Ba}$ in plasma of patients with Gram negative bacteraemia (GNB), Gram positive bacteraemia (GPB), rheumatoid arthritis (RA) and normal controls (NC). Values are expressed as percentage of the $\mathrm{Ba}$ standard. Horizontal bars represent the median values. GNB patients have significantly higher $\mathrm{Ba}$ values than controls $(p<0.01)$. Patients with GPB and rheumatoid arthritis have $B a$ values similar to those of controls. 
positive correlation was observed between the ESR and $\mathrm{Ba}$ values $(\mathrm{r}=0.49, \mathrm{p}<0.03)$.

\section{Discussion}

To assess complement activation via the alternative pathway we established a laser nephelometric technique that measures fragment $\mathrm{Ba}$. Significantly increased values of $\mathrm{Ba}$ were found in patients with Gram negative bacteraemia in which the alternative pathway is known to be activated. ${ }^{7}$ In contrast, $\mathrm{Ba}$ values were found to be normal in most of the patients with Gram positive bacteraemia and with rheumatoid arthritis. Our data show that when infection is suspected the finding of increased values of $\mathrm{Ba}$ implicates Gram negative micro-organisms.

Activation of the alternative pathway has previously been assessed by showing a reduction in the amount of the intact factor B molecule. ${ }^{7}$ This provides only indirect information concerning activation as extensive factor $B$ breakdown would be required to produce a measurable reduction in the factor $B$ molecule. $\mathrm{Ba}$ is generated by the enzymatic cleavage of factor B which occurs only when the alternative complement pathway is activated. ${ }^{5}$ The plasma concentration of $\mathrm{Ba}$, therefore, provides a specific indication of the state of complement activation via this pathway. To measure $\mathrm{Ba}$ directly this fragment must first be separated from larger factor B related molecules, then quantitated by the use of anti-factor B antiserum. The separation step is required as the polyclonal anti-factor B antiserum recognises antigenic determinants present both on the parent molecule and the split product.

An essay exploiting PEG separation of $\mathrm{Ba}$ has been described previously. ${ }^{12}$ The quantitation of the fragment was, however, performed using radial immunodiffusion, which requires $\mathbf{4 8}$ hours to produce results and is insensitive. Evidence of activation through the alternative pathway has also been provided by electrophoretic techniques. ${ }^{13}$ These techniques, however, are insensitive, give only qualitative results, and are unsuitable for analysis of large batches of samples. In contrast, laser nephelometry is sufficiently sensitive to detect $\mathrm{Ba}$ in healthy subjects and permits the automatised analysis of large sample batches in a short time.

In $56 \%$ of patients with Gram negative bacteraemia, $\mathrm{Ba}$ values were significantly increased, confirming the role of the alternative pathway. In a previous study ${ }^{7} 31 \%$ of patients showed a reduction in the values of factor B and properdin, when measured by a radial immunodiffusion technique. The greater number of patients showing evidence of activation of the alternative pathway in our study was probably a reflection of the higher sensitivity of our technique. Detection of increased amounts of the $\mathrm{Ba} \mathrm{O}$ fragment in patients with Gram negative bacteraemia $\stackrel{*}{*}$ suggests that this technique may be useful in the diag- 을 nosis and management of septicaemia. It has the advantage of providing results within five hours; $\stackrel{\vec{P}}{+}$ blood cultures require at least 24 hours' incubation. 을 The assay may be particularly useful in groups prone to infections but in whom the clinical diagnosis is $\frac{\bar{\omega}}{\vec{D}}$ difficult, such as neonates, the elderly, and the immu- $\stackrel{\varnothing}{\varrho}$ nosuppressed.

The results obtained in Gram positive bacteraemia suggest that the alternative pathway is not activated. In contrast to Gram negative bacteria, the cell walls $\vec{\omega}$ of Gram positive bacteria contain less lipo- $\stackrel{\circ}{\circ}$ polysaccharide ${ }^{14}$ and are therefore poor activators of $\frac{8}{8}$ the alternative pathway.

Our results indicate that in some patients with rheumatoid arthritis there is increased breakdown of $\circ$ factor B. Complement activation in rheumatoid $\vec{N}$ arthritis is known to proceed mainly along the classi- $\mathrm{G}$ cal pathway. ${ }^{9}$ The triggering of the alternative path- $\frac{}{2}$ way is influenced by the concentrations of $\mathrm{C} 3 \mathrm{~b} .{ }^{15} \mathrm{It}$ is thus conceivable that high concentrations of $\mathrm{C} 3 \mathrm{~b}$ pro- $\bigcirc$ duced in rheumatoid arthritis through the classical $\stackrel{\circ}{\circ}$ route initiate the activation of the alternative path- $\mathbb{D}$ way, ultimately leading to an increased breakdown of $\overrightarrow{\vec{O}}$ factor $\mathrm{B}$ and generation of $\mathrm{Ba}$ (fig 1). Interestinglg $\stackrel{\infty}{\infty}$ the extent of activation of the complement alternative pathway correlated significantly with the degree of disease activity, as assessed by the ESR.

The technique that we have developed may be useful in the management of high $\mathrm{Ba}$ values in patients with Gram negative bacteraemia, and in other condi- $\stackrel{\square}{\Omega}$ tions in which the alternative complement pathway $\overrightarrow{\vec{O}}$ has a role in the pathogenesis.

Dr G Senaldi is supported by Fondazione Anna Villa Rusconi, Varese, Italy. We are indebted to $\mathrm{Dr} M$ Nilsson, Dakopatts, Copenhagen, Denmark, for? helpful discussion and advice.

\section{References}

1 Ruddy S, Gigli I, Austen KF. The complement system of man. (Fourth of four parts). N. Engl J Med 1972;287:642-6.

2 Wicher JT. The value of complement analysis in clinical chemistry. Clin Chem 1978;24:7-22.

3 Lachmann PJ, Peters DK. Complement. In: Lachmann PJ, Peters $\mathrm{N}$ DK, eds. Clinical aspects of immunology. Oxford: Blackwell $\mathrm{N}$ Scientific Publications, 1982:18-49.

4 Lachmann PJ, Hughes-Jones NC. Initiation of complement acti- N vation. Springer Semin Immunopathol 1984;7:143-62.

5 Pangburn MK, Müller-Eberhard HJ. The alternative pathway of complement. Springer Semin Immunopathol 1984;7:163-92.

6 Brown EJ, Joiner KA, Frank MM. The role of complement in host resistance to bacteria. Springer Semin Immunopathol $\stackrel{\infty}{+}$ 1983:6:349-60.

7 Fearon DT, Ruddy S, Shur PH, McCabe WR. Activation of the properdin pathway of complement in patients with Gram nega- $\frac{\vec{D}}{D}$ . 
tve bacteraemia. N. Engl J Med 1975;292:937-40.

8 Zvaifler NJ. Rheumatoid synovitis: an extravascular immune complex disease. Arthritis Rheum 1974;17:297-305.

9 Lambert PH, Nydegger UE, Perrin LH, McCormick J, Fehr K, Miescher PA. Complement activation in seropositive and seronegative rheumatoid arthritis. 125-I-Clq binding capacity and complement breakdown products in serum and synovial fluid. Rheumatology 1975;6:52-9.

10 Krick EH, De Heer DH, Kaplan RA, Arroyave CM, Vaughan JH. Metabolism of factor B of serum complement in rheumatoid arthritis. Clin Exp Immunol 1978;34:1-9.

11 Vergani D, Bevis L, Nasslaruddin BA, Mieli-Vergani G, Tee DEH. Clinical application of a new laser nephelometric technique to measure complement activation. J Clin Pathol 1983;36:793-9.

12 Perrin LH, Lambert PH, Miescher PA. Complement breakdown products in plasma from patients with systemic lupus erythematosis and patients with membranoproliferative or other glomerulonephritis. J Clin Invest 1975;56:165-76.

13 Cooper NR, Nemerrow GR, Meyers JT. Methods to detect and quantitate complement activation. Springer Semin Immunopathol 1983;6:173-94.

14 Leive CL, Davis BD. Cell envelope; spores. In: Davis BD, Dulbecco R, Eisen HN, Ginsberg HS, eds. Microbiology. Hagerstown: Harper and Row, 1980: 71-110.

15 Vergani D. Complement. Diabetic Medicine 1986;3:306-11.

Requests for reprints to: Dr D Vergani, Department of Immunology, King's College School of Medicine and Dentistry, Denmark Hill, London SE5 8RX, London. 